\title{
New installation for inclined EAS investigations
}

\author{
E.A. Zadeba ${ }^{1, a}$, N.V. Ampilogov ${ }^{1}$, N.S. Barbashina ${ }^{1}$, A.G. Bogdanov ${ }^{1}$, A.A. Borisov ${ }^{1,2}$, D.V. Chernov ${ }^{1}$, L.I. Dushkin ${ }^{1}$, \\ R.M. Fakhrutdinov ${ }^{1,2}$, R.P. Kokoulin ${ }^{1}$, K.G. Kompaniets ${ }^{1}$, A.S. Kozhin ${ }^{1,2}$, V.V. Ovchinnikov ${ }^{1}$, A.S. Ovechkin ${ }^{1}$, \\ A.A. Petrukhin ${ }^{1}$, V.V. Shutenko ${ }^{1}$, N.S. Volkov ${ }^{1}$, V.S. Vorobjev ${ }^{1}$, and I.I. Yashin ${ }^{1}$ \\ 1 National Research Nuclear University MEPhI (Moscow Engineering Physics Institute), 115409 Moscow, Russia \\ 2 RF SSC Institute of High Energy Physics, Protvino 142281, Russia
}

\begin{abstract}
The large-scale coordinate-tracking detector TREK for registration of inclined EAS is being developed in MEPhI. The detector is based on multiwire drift chambers from the neutrino experiment at the IHEP U-70 accelerator. Their key advantages are a large effective area $\left(1.85 \mathrm{~m}^{2}\right)$, a good coordinate and angular resolution with a small number of measuring channels. The detector will be operated as part of the experimental complex NEVOD, in particular, jointly with a Cherenkov water detector (CWD) with a volume of 2000 cubic meters and the coordinate detector DECOR. The first part of the detector named CoordinateTracking Unit based on the Drift Chambers (CTUDC), representing two coordinate planes of 8 drift chambers in each, has been developed and mounted on opposite sides of the CWD. It has the same principle of joint operation with the NEVOD-DECOR triggering system and the same drift chambers alignment, so the main features of the TREK detector will be examined. Results of the CTUDC development and a joint operation with NEVOD-DECOR complex are presented.
\end{abstract}

\section{Introduction}

The aim of the large-scale coordinate-tracking detector is solving the problem of the excess of muon bundles that increases with the energy of the primary cosmic rays $[1,2]$, that can be caused by both cosmo- or nuclear-physical reasons [3]. The only characteristic that responds differently to changes in the composition of cosmic rays and the inclusion of new physical processes is the energy of the muon component of extensive air showers [3] which, up to now, has not been investigated enough. Such studies are performed at the experimental complex NEVOD-DECOR [4]; however, the coordinate detector DECOR does not cover the entire aperture of the Cherenkov water detector (CWD) and does not exclude the possibility of several muons passing between the individual supermodules of the detector. Besides, the size of its cells limits the possibility of separating two or more particles at small distances (less than $3 \mathrm{~cm}$ ). The new coordinate-tracking detector [5] based on drift chambers will increase the coverage of the side aperture of the Cherenkov water detector NEVOD and significantly improve the resolution of close tracks.

\section{Drift chambers}

Drift chambers (DC) came to accelerator particle experiments and cosmic ray studies in the late 70-ies of the last century, after proportional chambers. Their main advantage is the ability to ensure the measurement accuracy of charged particle tracks significantly better than the characteristic distance between sensitive elements (signal wires). This property provides the possibility to

a e-mail: EAZadeba@mephi.ru create large-scale installations. One such setup created in the 80-ies was the IHEP-JINR neutrino detector at the U-70 accelerator, for which a large area multiwire drift chamber was developed [6].

Overall sizes of the chambers are $4000 \times 508 \times$ $112 \mathrm{~mm}^{3}$ with a sensitive area of $1.85 \mathrm{~m}^{2}$ that is $91 \%$ of the side chamber area. There are four sense wires in the middle of the chamber alternately shifted by $\pm 0.75 \mathrm{~mm}$ parallel to the drift direction to solve right-left ambiguity. The distance between the sense wires is $10 \mathrm{~mm}$. There are two guard wires to remove the edge effect. The sense and guard wires are surrounded by the cathode wires. A uniform electric field is created in the drift gap by fieldforming wires with a uniformly distributed potential. The chamber gas volume is limited by the aluminium alloy case, $1.5 \mathrm{~mm}$ thick, which serves simultaneously as an electric screen and the chamber frame. The chamber ends are limited by plexiglass plugs, where wires, gas inlet and outlet, amplifiers, high voltage supply circuit and cable connectors are assembled. Blind holes in the plugs are provided for the installation of the drift chambers so they are firmly isolated from the setup framework. Due to a nice uniformity of the electric field inside the chamber, electron drift velocity can be assumed to be constant and we can use a linear relation between drift time and coordinate. Configuration of signal wires enables reconstruction of a projection of particle track to the plane orthogonal to the wires. Thus, for reconstruction in space at least two non-parallel drift chambers are required. The chamber is filled with a gas mixture of $\mathrm{Ar}(94 \%)$ and $\mathrm{CO} 2(6 \%)$ at a small overpressure of about 10-20 mbar. The spatial accuracy of the IHEP drift chamber is $0.6 \mathrm{~mm}$ and its angular resolution is about 0.03 rad. Right-left ambiguity is resolved in $98 \%$ of events. 

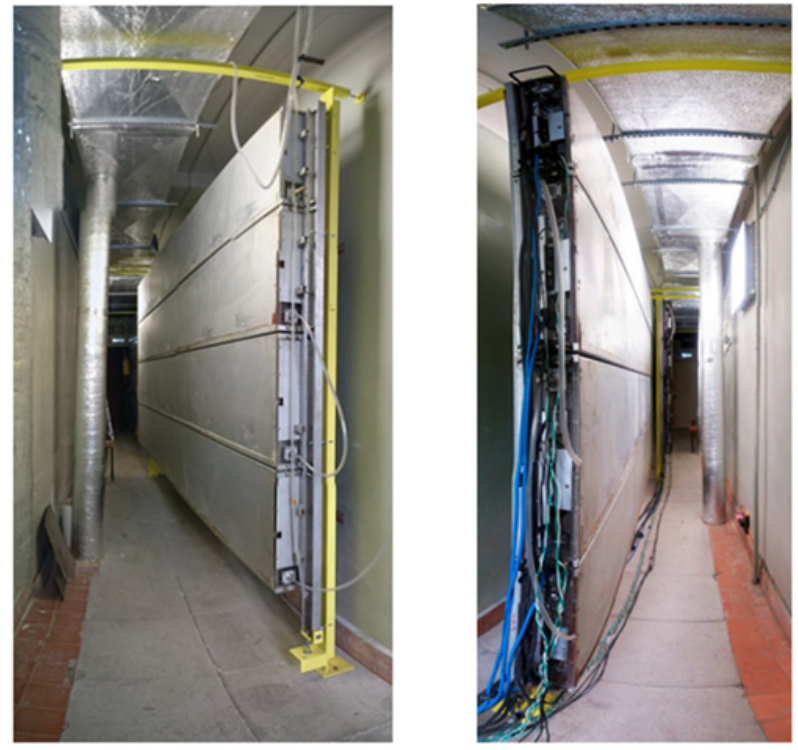

Figure 1. Photographs of the CTUDC planes.

The signals from the wires are processed by an on-board amplifier-shaper that forms 75-100 ns pulses (depending on the input signal) in the LVDS levels.

\section{CTUDC}

\subsection{Placement}

The coordinate-tracking unit based on drift chambers (CTUDC) consists of two vertical coordinate planes installed at different sides of the CWD in the short galleries of the third floor of the NEVOD building, one floor above the DECOR supermodules (Fig. 1). Such a location allows the registration of near horizontal tracks by CTUDC (triggered by CWD) or also by joint operation with DECOR, that will significantly increase the range of muon track zenith angles from $85^{\circ}-95^{\circ}$ to $80^{\circ}-100^{\circ}$.

Each plane consists of 8 drift chambers installed in two rows, overlapping by $30 \mathrm{~cm}$ to exclude dead zones in the chamber ends; it causes a $4^{\circ}$ angle between the planes and CWD wall. The chambers are mounted on a special frame (Fig. 1) that allows precise adjustment of the DCs in all directions.

The distance between the registration system of the setup and the chambers is different for planes. It causes the difference in the length of cables and requires additional commutation blocks. The effective area of the plane is $14.8 \mathrm{~m}^{2}$, the area of two DECOR supermodules located a floor below is $17.5 \mathrm{~m}^{2}$, so the total area of the coordinate detectors for registration of near-horizontal particles coming along the CWD almost doubles.

\subsection{Power supply}

The drift chambers are supplied with two high voltages: $12 \mathrm{kV}$ (field-forming wires) and $2.2 \mathrm{kV}$ (signal wires), and with $\pm 6 \mathrm{~V}$ low-voltage. The high voltage is provided by the multi-channel HV power supply controlled by a PC via a USB bus. The software of the CTUDC controls the current on the wires providing a smooth rise of the voltage during turning on the supply. A special microcontroller gets commands from software and turns on a relay to

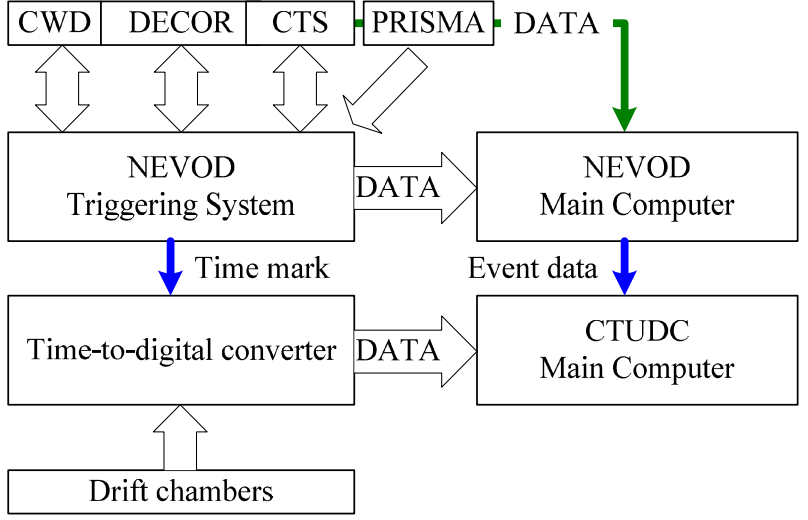

Figure 2. Principal scheme of the joint operation of CTUDC and NEVOD TS.

enable a light alarm outside the gallery of CTUDC. The most dangerous process is turning on/off $12 \mathrm{kV}$, since it causes electrostatic potential on the chambers. The light alarm flashes at $1 \mathrm{~Hz}$ during such process until the current stops rising (it always glows at a steady voltage). Such capabilities of the supply allow getting reliable noise characteristics of the chambers, because every point in such a measurement should be obtained with a steady current [7].

Two linear low-voltage supplies provide $\pm 6 \mathrm{~V}$ to amplifiers situated on the DCs, their total consumption is about $6 \mathrm{~A}$.

\subsection{Gas supply}

The gas preparation system is situated on the first floor of the building. Iit consists of two gas tanks $\left(\mathrm{Ar}\right.$ and $\left.\mathrm{CO}_{2}\right)$, ramp, two controlled flow meters, gas mixer and the PC, which manages the modes of gas supply. The mixture is supplied to the chambers via PVC tubes. Software of the system allows tracking of volumes of gas passing through chambers and to turn off the flow if one component of the mixture is exhausted. The CTUDC has a sequential gas tube connection, but every chamber or a group of chambers can be connected separately to the gas system.

\subsection{Registration system}

CTUDC is designed for joint operation with the triggering system of the experimental complex NEVOD (NEVOD TS) that binds registration systems of CWD, DECOR, calibration telescope system (CTS) and array of neutron detectors PRISMA. The triggering system has a rather fast data handling: the period between the passage of a particle through the working volume of CWD and the trigger formation is about $500 \mathrm{~ns}$. On the other hand, the maximum drift time of electrons in the drift chamber is $6 \mu \mathrm{s}$, so the registration system and DAQ of CTUDC cannot be directly integrated into the NEVOD TS and should be implemented separately with the possibility of off-line inter-connection between the NEVOD and CTUDC data.

The registration system of CTUDC (Fig. 2) consists of a CTUDC main PC (MPC), VME crate with an optical bridge and a 128-channel time-to-digital converter (TDC) CAEN V1190. The TDC resolution is 100 ps while the time resolution of the drift chambers is about $5 \mathrm{~ns}$. The 


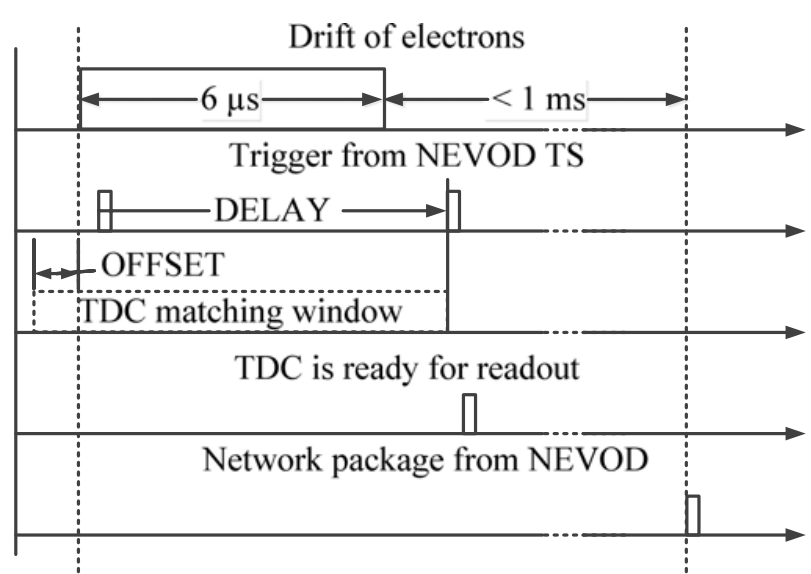

Figure 3. Timing diagram of the joint operation of CTUDC and NEVOD TS.

internal memory of the device allows storing large amount of events to the moment of TDC readout. The ability to work in a continuous mode allows measuring signal and noise rate per-channel with more than $90 \%$ live time.

The timing diagram of the CTUDC registration system is shown in Fig. 3. The NEVOD TS fixes an event if one of the experimental complex setups is triggered: CWD, DECOR, CTS and PRISMA. Each setup has its own trigger conditions. For CWD it is lighting up more than 60 measuring modules [8], for DECOR it is triggering at least two supermodules. For CTS there are three conditions: triggering of several detectors of the top plane, triggering of several detectors of the bottom plane, triggering of a single detector in each plane. For PRISMA it is triggering of two neutron detectors by charged particles (the threshold corresponds to the simultaneous passage of five particles within the scintillator detector). The NEVOD TS gathers information from all setups for every event, no matter if they gave no trigger.

In the main mode of operation, the trigger for CAEN TDC should be received after all hits of the event, so the signal from NEVOD TS is delayed for $8 \mu$ s. The matching window of TDC is chosen to be certainly longer than the delay value, so it is $12 \mu \mathrm{s}$. This leads to the fact that the starting point of counting the electron drift time is different from zero in the TDC data. The position of that point in the data is called the offset. In fact, it consists of two parts: basic offset for all channels $(\sim 1.5 \mu \mathrm{s})$ and individual offset caused by the difference in the length of the signal wires of the right and left planes of CTUDC and different twisting step in them.

For several types of events, MPC of NEVOD sends a network packet via Ethernet with information about the last event for further matching of CTUDC and NEVOD TS data, the most important information in the packet is the event number.

\subsection{Software}

CTUDC software is divided into server and client programs. The first one is constantly running on a MPC of CTUDC, it provides exposition, controls VME crate with TDC, HV supply and light alarm. The client can be launched on multiple computers in a shared server network; it can control modes of the exposition and the high voltage supply. It is also used as a program for remote monitoring of the setup. Communication between applications is held via UDP TCP/IP Protocol in a synchronous mode. Conflicts between client programs for the server time were not observed.

Because of the relatively high frequency of events in the NEVOD TS, network packets cannot be sent for each event that could result in an overload of the internal network, but only for two types of events: triggering of one supermodule DECOR in each short gallery, and triggering of CWD and DECOR at the same time. The total frequency of such events is around $1 \mathrm{~Hz}$. After the reception of the network packet a readout of TDC events occurs. Then the server software compares the number of read events with the difference in the event numbers in the last two network packets. If these numbers match, then all read events are assigned with a corresponding intermediate number. Otherwise, this set of events is logged in a special file for individual processing. It happens on average to $4 \%$ of events.

Currently, a new mode of exposition is being tested: when the difference in numbers is found the system waits for the third network packet and then the comparison is performed for the events between the first and the third package. This reduces the number of dropped events to $0.5 \%$.

Every 20 minutes, NEVOD is monitored for 30 seconds, the MPC of CTUDC receives a network packet at the beginning of this process and software starts the 30 seconds measurement of noise from drift chambers. The results are stored on the server that allows to monitor the performance of the DCs and to make corrections for specific pieces of data.

\section{Processing of CTUDC data}

Primary processing of CTUDC data includes calibration of drift chambers (forming accurate configuration files for further processing) and passportization of experimental runs.

\subsection{Calibration}

The calibration of the unit is performed after every replacement of drift chambers and amplifiers in them. It includes the correction of data, the position of the drift chambers and the selection of individual offsets for the measuring channels.

A spatial calibration is performed by cross-calibration of CTUDC with DECOR supermodules [9]. The position of the drift chambers is varied by software until the position corresponding to the best matching of tracks in both systems will be found.

The determination of individual offsets for each wire is performed according to calibration series. Statistics for one channel in such a run is about 100000 hits. Figure 4 shows the distribution of hits in drift time per channel. The background of the distribution is due to noise; the duration of the main part of the distribution is 5.5-6 $\mu \mathrm{s}$, which corresponds to the maximum drift time of the electrons and depends on the current state of the gas mixture in the chamber. The position of the left edge of the distribution corresponds to the individual offset of the channel. However, this definition cannot be considered absolutely reliable because of the rather large value of bins 


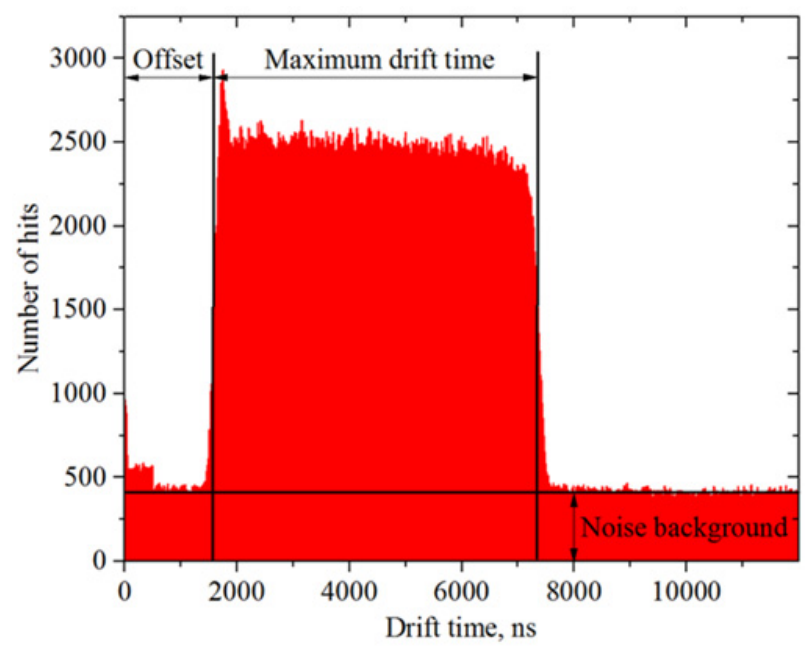

Figure 4. Distribution of hits in drift time.

(about $10 \mathrm{~ns}$ ), and due to the large influence of the jitter of the receipt of the trigger signal. Therefore, precision accuracy in the value of the individual offset is determined by a sequential search method.

For each chamber about 10,000 single tracks are selected to a separate file. Individual offsets of the channels are varied in increments of $2.5 \mathrm{~ns}$ relative to each other and for each combination software makes the reconstruction of all tracks using the least squares method. The algorithm chooses the combination in which the number of tracks with the sum of squares of deviations of the track from the experimental points is less than a pre-selected threshold $\left(10 \mathrm{~mm}^{2}\right)$ is maximized. The basic offset is obtained by the same method, but it uses events nearly perpendicular to the chamber plane, the criterion is smoothness of the distribution of the coordinate of intersection of reconstructed track with the drift chamber plane. If the offset is chosen incorrectly, the peak or a gap will occur in the area corresponding to the center of the chamber in such distribution.

\subsection{Determination of current chamber characteristics}

The value of the drift velocity is determined individually for each chamber in each experimental run and is texted in the passport of the given run. Not only is the width of the distribution of the drift time important, but also its form. During degradation of the gas mixture the distribution of the hit drift times (Fig. 4) changes its form: it falls in the region of large drfit times and looks trapezoidal, i.e., the efficiency of the chamber drops for the registration of particles that have passed far from the center of the chamber. Such defective chambers should be flushed with 600 liters of the gas mixture. The average time of operation of the chamber without additional venting is about 3 months.

One of the most important characteristics of the drift chambers is the efficiency of the signal wires. Efficiency is defined as the ratio of the number of events without the participation of the signal wire to the sum of this value with the number of full events (with all triggered wires) in DC. It was $99.95 \%$ at the test bench.
In the experiment, this value is $99.3 \%$ on average. The decrease in the efficiency is due to the increase of the threshold shaper-amplifiers, which was required because of a higher level of electromagnetic interference in the CTUDC in comparison with a test bench. The threshold level for the amplifiers is set so that the noise rate on the chamber channels does not exceed $2 \mathrm{kHz}$.

\subsection{Passportization}

To handle joint events of CTUDC and NEVOD TS the offline linking of events is carried out. Joining is made in several stages. The first step is to separate the CTUDC data on the parts corresponding to the experimental runs of the NEVOD TS (approximately 40 hours of exposition). Then DECOR tracks are reconstructed in NEVOD data. The third stage is forming of a single file with the events that have a structure given in Table 1 .

Table 1. Format of joint events.

\begin{tabular}{|l|l|}
\hline Statistics of the NEVOD RUN \\
\hline Header of the event \\
\hline CTUDC & RUN and event number, time \\
\cline { 2 - 2 } & Number of hits \\
\cline { 2 - 2 } & $\begin{array}{l}\text { Triple vector of hits } \\
\text { (Chamber : Wire : Time) }\end{array}$ \\
\cline { 2 - 2 } & Event statistics \\
\hline NEVOD & RUN and event number, time \\
\cline { 2 - 2 } & $\begin{array}{l}\text { Number of trigger signals from CWD } \\
\text { and CTS }\end{array}$ \\
\cline { 2 - 2 } & $\begin{array}{l}\text { Number of modules with amplitude } \\
\text { information }\end{array}$ \\
\cline { 2 - 2 } & Status registers of TS \\
\cline { 2 - 2 } & Pressure, temperature, etc. \\
\hline DECOR & Number of tracks \\
\cline { 2 - 2 } & $\begin{array}{l}\text { Vector of track information } \\
\text { (Vertex : Vector) }\end{array}$ \\
\hline$\ldots$ & \\
\hline RUN trailer & \\
\hline
\end{tabular}

The passportization of the experimental runs is carried out after joining. The passport includes information about the correspondence of events, efficiency of the measuring channels and statistics on the frequency of different classes of events in CTUDC. The drift velocities are also specified and are later used in event handling.

\section{First results}

For the moment, more than 2500 hours of debugging and measuring series have been held at the CTUDC. According to their data, a cross-calibration was made with the coordinate-tracking detector DECOR and the individual offsets for the measuring channels were determined.

Figure 5 shows the distribution of events according to the setups that triggered NEVOD TS. The graph shows that the least likely response in CTUDC is accompanied by events from CTS and DECOR (usually these are single muon events). Most of the events from PRISMA are usually accompanied by CTUDC response. Figure 6 shows the same distribution for the average number of triggered events in DC at different trigger types. Events of the PRISMA setup typically have a greater density of charged 


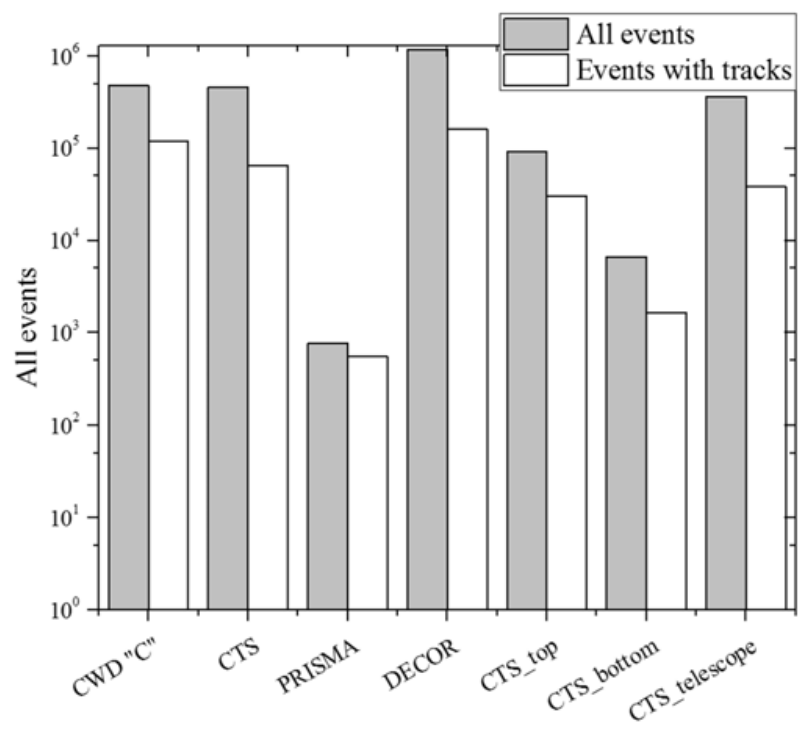

Figure 5. Number of events per each trigger for one experimental run.

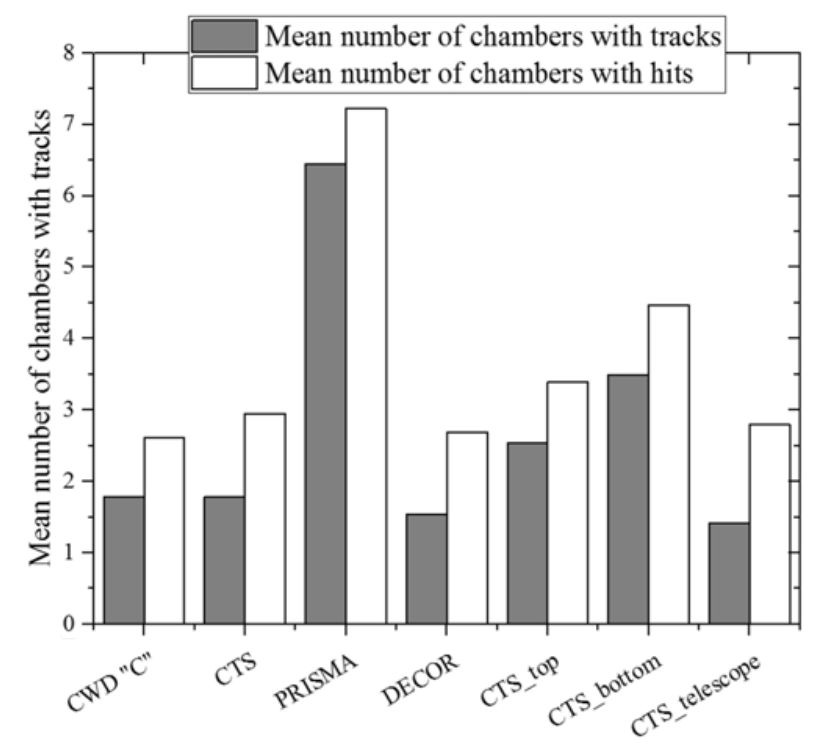

Figure 6. Mean number of hit chambers in events with different triggers.

particles (due to a high threshold on the detectors). In such events, on average, up to 7 drift chambers have tracks. On the other hand, events with CTS trigger are characterized by a large difference between the number of chambers with full tracks and chambers having at least one hit. This suggests that most of the hits on this trigger are accidental (noise).

The setup is able to register single muons and muon bundes with density up to 10 particles per $\mathrm{m}^{2}$. Figure 7 shows the distribution of registered events in track multiplicity.

Processing of the data uses three methods of reconstruction of multiple events: the sequential search method, the method of finding the straight line and the histogram method. The last method determines the number of tracks under the same zenith angle in the event. It is most

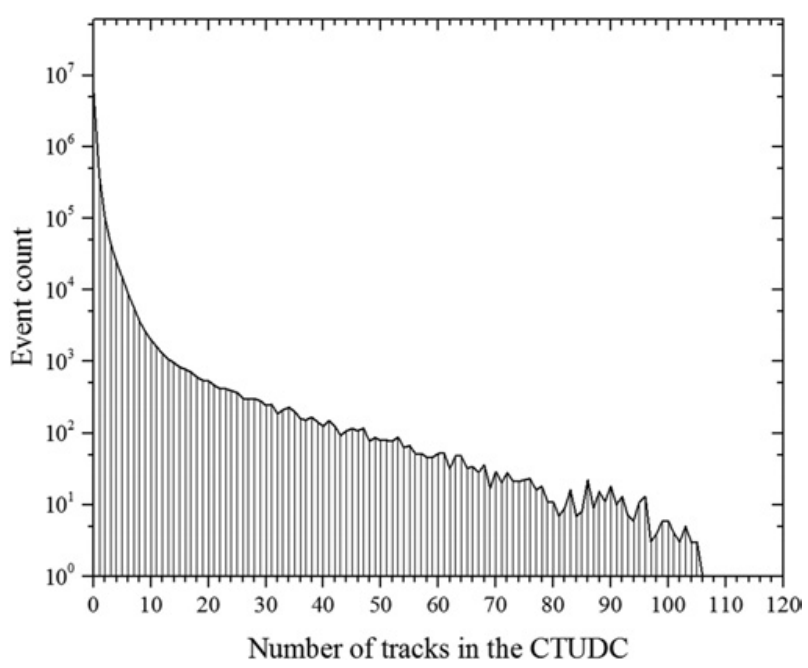

Figure 7. Distribution of multiplicity of events in CTUDC.

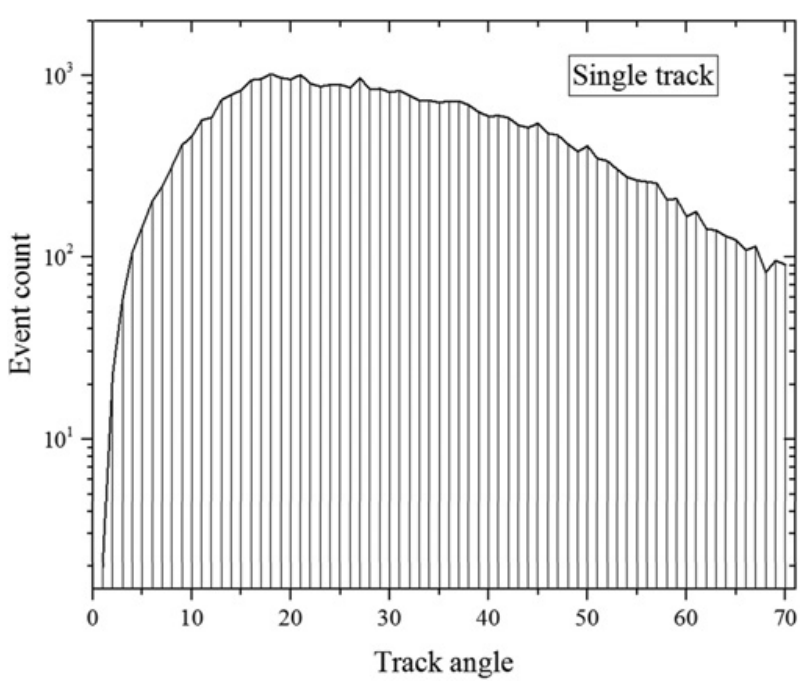

Figure 8. Distribution of single track events in the angle of their projections inside the drift chamber.

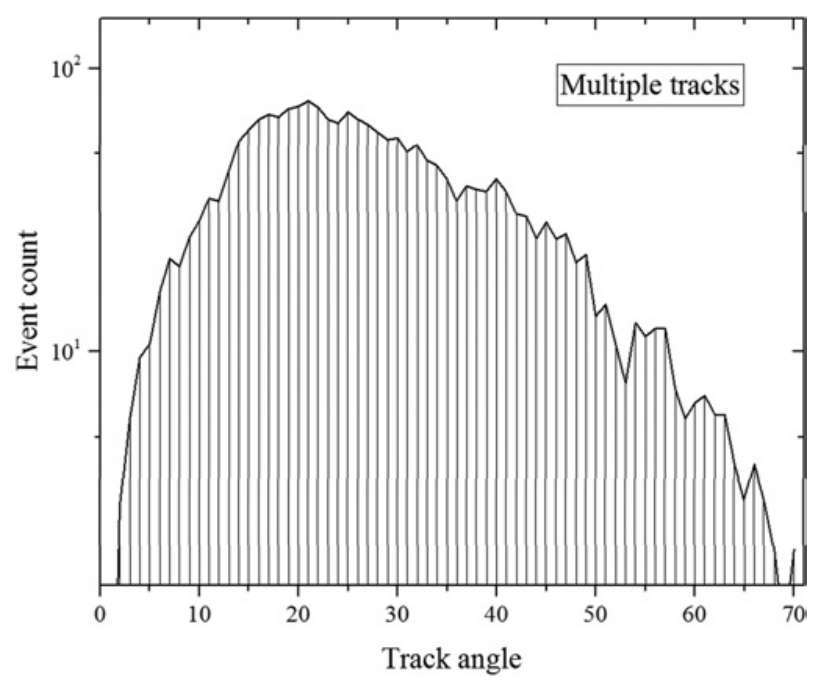

Figure 9. Distribution of multiple track events in the angle of their projections inside the drift chamber. 
suitable for the determination of multiplicity: because of the remoteness of the points of generation of muons, their trajectories are quasiparallel. The influence of the dead time of measuring channels $(\sim 100 \mathrm{~ns})$ and the number of secondary particles produced in the concrete walls of the NEVOD building are also taken into account in determining the number of particles in the bundle.

Figure 8 shows the distribution of events according to the value of the angle between the projection of the track on the plane othogonal to signal wires and a plane passing through them. The $90^{\circ}$ angle corresponds to vertical tracks, the zero angle corresponds to tracks with zenith angle of $90^{\circ}$ and perpendicular to the DC plane.

Figure 9 shows the same distribution for multiple events. Both distributions have maxima in the region of $20^{\circ}$, that is due to the fact that the events are selected according to the trigger from CWD and DECOR so it means that particles moved horizontally through the chambers to the water volume or in the opposite direction.

\subsection{Conclusion}

The new coordinate-tracking unit on the drift chambers (CTUDC) is developed in MEPhI. The installation greatly improves the capabilities of the experimental complex
NEVOD in the registration of near-horizontal extensive air showers.

The setup successfully passed adjustments and calibration stages and is currently gathering experimental data.

\section{References}

[1] N.S. Barbashina et al., Nucl. Phys. B (Proc. Suppl.) 165, 317 (2007)

[2] A.G. Bogdanov et al., Physics of Atomic Nuclei 73, 1852 (2010)

[3] A.A. Petrukhin, Nucl., Phys. B (Proc. Suppl.) 212213, 228 (2011)

[4] E.A. Kovylyaeva et al., Bull. Lebedev Phys. Inst. 41, 218 (2014)

[5] E.A. Zadeba et al., JINST 9, C08018 (2014)

[6] N.I. Bozhko et al., Nucl. Instrum. Meth. A 243, 388 (1986)

[7] E.A. Zadeba et al. J. Phys.: Conf. Ser. 632, 012031 (2015)

[8] O. Saavedra et al., J. Phys.: Conf. Ser. 409, 012009 (2013)

[9] E.A. Zadeba et al. J. Phys.: Conf. Ser. 675, 032039 (2016) 